had no effect. Other reflex epilepsies induced by higher mental activity include reading epilepsy, graphogenic epilepsy induced by writing, language induced epilepsy induced by reading, writing, and speaking, epilepsia arithmetices induced by calculation, decision making epilepsy induced by cards and game playing, and drawing induced seizures induced by geometric designs. Nintendo and Rubik's cube may also cause reflex epilepsy.

\title{
CORPUS CALLOSOTOMY FOR INTRACTABLE SEIZURES
}

The results of corpus callosotamy in 18 patients 16 years old and younger are reported from the Sections of Neurology and Neurosurgery, Dartmouth-Hitchcock Medical Center, Hanover, NH. A significant improvement with a decrease in seizure frequency of greater than $80 \%$ occurred in $83 \%$ of patients having generalized atonic, tonic, or tonic-clonic seizures. There was no postoperative deterioration in behavior, memory, or language function when the callosotony was performed as a two stage procecure. Some patients had a very mild and transient hemiparesis that resolved in a few days but none developed persistent mutism, a reported complication in other series. Many generalized seizures were converted to focal seizures postoperatively. Partial seizures are usually not responsive. Patients with evidence of unilateral disease tend to have the best results from callosotomy while those with generalized infantile spasms, Lennox-Gastaut syndrome, or anoxic encephalopathy have less predictable results. Mental retardation is not a contraindication and improvement in control of the seizures may improve behavior and cognitive functioning. (Nordgren RE et al. Corpus callosotmy for intractable seizures in the pediatric age group. Arch Neurol April 1991; 48:364-372).

COMMENT. Callosotomy may be considered in young patients with frequent generalized atonic, tonic, and tonic-clonic seizures. Early surgical intervention may prevent the adverse effects of frequent recurrent seizures and the possibility of kindling.

\section{CORTIOOSTEROIDS FOR LANDAU-KLEFFNER SYNDROME}

Four children between five and nine years of age with Landau-Kleffner syndrome were treated with ACTH or corticosteroids at the Department of Pediatrics, Beilinson Medical Centre, and Sackler Faculty of Medicine, Tel Aviv University, Israel. ACTH in one patient was given for three months starting with 80 units/day followed by a gradual reduction of the dose. An EEG three weeks after the start of the treatment showed complete disappearance of epileptic activity but the aphasia was initially unchanged. Complete remission of the aphasia occurred eight months after the completion of the ACIH course. Two years later the aphasia recurred with the concomitant appearance of multiple spike discharges on the EEG. Prompt prescription of ACTH therapy led to a recovery of speech and a normal EEG within a few weeks. Prednisone $60 \mathrm{mg} /$ day for two to three months in two patients caused a prompt improvement in the EEG followed by normal speech. Dexamethasone $4 \mathrm{mg} /$ day resulted in full recovery of speech and a 